\title{
Characterization of a novel LEDs device prototype for neonatal jaundice and its comparison with fluorescent lamps sources: Phototherapy treatment of hyperbilirubinemia in Wistar rats
}

\author{
Priscilla Fróes Sebbe ${ }^{\mathrm{a}}$, Antônio Balbin Villaverde ${ }^{\mathrm{b}}$, Leonardo Marmo Moreira ${ }^{\mathrm{b}, \mathrm{c}, *}$, \\ Ana Maria Barbosa ${ }^{\mathrm{a}}$ and Nelson Veissid ${ }^{\mathrm{d}}$ \\ ${ }^{a}$ Instituto de Pesquisa e Desenvolvimento, Universidade do Vale do Paraíba, São José dos Campos, SP, \\ Brazil \\ ${ }^{\mathrm{b}}$ Universidade Camilo Castelo Branco, São José dos Campos, SP, Brazil \\ ${ }^{\mathrm{c}}$ Departamento de Engenharia de Biossistemas, Universidade Federal de São João Del Rei, São João \\ Del Rei, MG, Brazil \\ ${ }^{\mathrm{d}}$ Laboratório Associado de Sensores e Materiais, Instituto Nacional de Pesquisas Espaciais, São José \\ dos Campos, SP, Brazil
}

\begin{abstract}
This work describes experimental tests done using the LED-therapy equipment developed for neonatal jaundice treatment. Neonatal phototherapy consists in the irradiation of jaundice new-born with a high intensity light, in the spectral range of 400-500 nm, in order to promote the photochemical transformation of the bilirubin molecules in the areas exposed to the light. A convenient light source for jaundice treatment can be obtained employing blue LEDs, which are simple devices that present low cost, small heating production, narrow spectral band and long life-time. Equipment using an array with 88 blue LEDs (emission peak at $472 \mathrm{~nm}$ with $50 \mathrm{~nm}$ bandwidth) was constructed, optically characterized and then validated in comparison with a commercial equipment of phototherapy, i.e., the Fluorescent Conventional. For the device validation, experiments were undertaken employing male Wistar rats, to which the hyperbilirubinemia was induced by the obstruction of the billi duct. Using the LED-therapy equipment, it was obtained a reduction by $78 \%$ of the indirect bilirubin concentration against only $39 \%$ referent to the commercial Fluorescent Conventional, both for $8 \mathrm{~h}$ of treatment. For a period of $24 \mathrm{~h}$, the reduction was $92 \%$ and $76 \%$, respectively. Thus, the novel LED-therapy equipment was more efficient for photodegradation of the bilirubin molecules in animal model, when compared with the Conventional equipment. The novel prototype present requisites to be applied in clinical treatments, such as a large and uniform area of irradiation, efficient wavelength emission for jaundice treatment, high spectral irradiance, simplicity and low values of weight, heating and cost. Therefore, the new device is candidate to systematic applications in phototherapy treatments.
\end{abstract}

Keywords: LEDs device prototype, phototherapy, hyperbilirubinemia, common-bile-duct obstruction

\footnotetext{
*Corresponding author: Leonardo Marmo Moreira, Universidade Camilo Castelo Branco (UNICASTELO), Rod. Presidente Dutra Km 138, Eugênio de Melo, CEP: 12247-004, São José dos Campos, SP, Brazil. E-mail: leonardomarmo@gmail.com.
} 


\section{Introduction}

Phototherapy is a treatment that consists in irradiating a patient with light of high intensity, which promotes beneficial photochemical transformations in the irradiated area. Indeed, due to the interaction between the biological tissue and the photons from a light source, which can present mono- or polychromatic character, a series of biochemical reactions occurs in the biological medium as consequence of the initial photochemical process, generating, frequently, chain reactions. The phototherapy for neonates is applied in order to break down the bilirubin molecules, which is an organic pigment originated from erythrocytes degradation, aiming to increase its excretion by the organism. In fact, it is well known that an elevated concentration of bilirubin in the neonate blood constitutes a pathology called "neonatal jaundice", which is associated to significant risk of death to the babies. The first clinical application of the neonatal phototherapy was reported by Cremer et al. in 1958 [9]. These authors observed that the bilirubin concentration in blood was reduced when the neonates were irradiated with light and, then, they constructed the first phototherapy system employing blue fluorescent lamps [9].

The neonatal jaundice control can be achieved by two methods: Blood transfusion or irradiating the neonate with light. A comparative study between these two methods was reported by Maisels [17]. The basic mechanism for the phototherapy action is the conversion of bilirubin molecules into hydro-soluble and less toxic metabolic forms. Bilirubin molecules absorb light in the blue region of the spectrum, i.e., in the spectral range between 400 and $500 \mathrm{~nm}[11,12]$. The electromagnetic radiation associated to this spectral region can penetrate the epidermis, reaching the subcutaneous tissue, being, consequently, absorbed by the bilirubin present in the blood. Because of the light attenuation in the biological tissue, only bilirubin molecules up to a depth of $2 \mathrm{~mm}$ from the skin will be irradiated by the light. In the literature, it was reported that the minimum spectral irradiance therapeutic dose is approximately of $4 \mu \mathrm{W} / \mathrm{cm}^{2} / \mathrm{nm}$ [17]. Nevertheless, it is recommended a spectral irradiance of, at least, $16 \mu \mathrm{W} / \mathrm{cm}^{2} / \mathrm{nm}$, since the efficiency of the phototherapy is strictly dependent of the light intensity irradiated on the respective biological area.

Following Cremer's work [9], several other studies on the efficacy of phototherapy using blue fluorescent lamps have been reported [1,2,5,11,12,16,17,24-28]. A new light source employing blue light emitting diodes (LEDs) was introduced in 1998 for neonatal jaundice treatment [31]. Since then, various studies have been reported in the literature regarding the efficacy of using LEDs for jaundice treatment and comparing its performance to other light sources, such as fluorescent light and halogen-quartz lamps $[6,19,21,22,29,30]$.

LEDs are simple, small and low cost devices. These devices operate with a low voltage between 1.6 and $3.3 \mathrm{~V}$ and the power consumption stands in the $10-150 \mathrm{~mW}$ region. LEDs present a narrow spectral emission band of approximately $50 \mathrm{~nm}$, which can overlap the absorption spectrum of the bilirubin. Some other advantages of using LEDs are the low heating and their long lifetime, well over $100,000 \mathrm{~h}[18]$.

In this context, the development of new prototypes of light sources that can improve the efficiency of several clinical treatments, such as neonatal jaundice treatment, constitutes a relevant contribution to increase the efficacy of the phototherapy, favoring a more significant popularization of this procedure and, for consequence, the cure of several children. For this reason, a new phototherapy device for neonatal jaundice treatment was developed by our research group, using as a light source an array of LEDs emitting in the blue region of the spectrum. The unit was constructed in agreement to the international 
norm IEC 60601-2-50, which establishes rules on safety and performance of phototherapy equipments for treatment of neonatal jaundice [20].

Bilirubin $\left(\mathrm{C}_{33} \mathrm{H}_{36} \mathrm{NaO}_{6}\right)$ is a pigment that, normally, is present in the organism in small quantities $[14,23]$. The hemoglobin consists in the principal biological source of bilirubin through the breakage of "adults" erythrocytes, which contributes with $80-85 \%$ of the total bilirubin production. The second origin of bilirubin, which corresponds to $15-20 \%$ of its total concentration, is due to the premature destruction of erythrocytes. Subsequently, the heme group is converted in carbon monoxide (CO) and biliverdin, which, in its time, is transformed in bilirubin $[3,14,15,23]$. This bilirubin that presents apolar characteristics is called "non-conjugated bilirubin" or, simply, "indirect bilirubin". After its transport by albumin through blood circulation until the liver, a phenomenon of conjugation occurs in the hepatocytes, originating the hydro-soluble bilirubin, which is also known as "conjugated bilirubin" or "direct bilirubin" $[3,14,15,23]$.

The phototherapy action transforms the bilirubin in hydro-soluble compounds, which are easily excreted by liver and kidney. Two photochemical mechanisms have been proposed in order to explain the phototherapy action in the reduction of bilirubin serum levels: photoisomerization, which is considered the most important phototherapic mechanism of bilirubin degradation, and photooxidation $[8,23,24]$. Photoisomerization is associated to the conversion of bilirubin in two isomers, which are known as geometric and structural isomers ("lumirubin"). The formation of structural isomer, called "lumirubin", is slower than the production of the geometric isomer; however, the generation of lumirubin is considered irreversible and, since this isomer presents high hydro-solubility, can be eliminated through the bile and urine, without any need of conjugation. Photooxidation mechanism, in its time, consists in the oxidation of bilirubin molecules in aerobic environment to generate hydro-soluble pirrolic derivatives, which are eliminated from organism through the urine. Nevertheless, photooxidation mechanism seems to be small representative in the decrease of the bilirubin serum levels when compared with the photoisomerization process $[8,23,24]$.

The present work is focused on the characterization and validation of a prototype of phototherapy equipment for jaundice treatment developed in our laboratory. Before clinical application of our novel device in jaundice neonates, animal experimentation was undertaken in which hyperbilirubinemia was induced in rats.

The bilious obstruction in rats through the ligation technique of the common bilious duct (LCBD) consists in a experimental model widely employed for the study of cholestatic illness and hyperbilirubinemia processes $[4,7]$. Jaundice is one of the clearest consequences of the LCBD, which is a frequent phenomenon when the hyperbilirubinemia reaches $3.5 \mathrm{mg} / \mathrm{dl}$ [13]. In fact, hystopathological and biochemical alterations in rats with bilious obstruction by LCBD had been well described in several works published in the literature $[4,7]$.

In this way, in order to characterize and validate our phototherapy equipment, two set of tests were undergone. Firstly, measurements focused on the emitted spectral irradiance as well as the mapping of the radiation spatial distribution was developed. Subsequently, it was elaborated a consistent study comparing our new prototype with a conventional commercial equipment, which makes use of fluorescent lamps. These analyzes were made undertaking procedures of animal experimentation in Wistar rats with hyperbilirubinemia induced by the LCBD technique. The experimental results obtained in the present work will be discussed in details in agreement with recent data from literature. 


\section{Materials and methods}

\subsection{Equipment description}

The optical device consists in an array with 88 blue LEDs ( 8 rows by 11 columns), connected electrically in a parallel configuration. The array was mounted onto a blanket made of antiallergenic, antimoldy and biocompatible foam. The blanket was constructed in a rectangular format $(20 \times 14 \mathrm{~cm})$, which corresponds to the medium size of a neonatal patient. In this way, all the patient body area would be irradiated. LEDs model OSUB5131P from Optosupply (China), which presented a diameter of $5 \mathrm{~mm}$, were employed. The optical characteristics of this device includes $50 \%$ power angle of $30^{\circ}$, emission wavelength centered at $472 \mathrm{~nm}$ with a narrow band-pass of $50 \mathrm{~nm}$ of width (Full Width at Half Maximum) and a luminous intensity of $6,250 \mathrm{mcd}$. The LEDs array was powered by a $5 \mathrm{~V}$ power supply, with an electric current intensity of 0.22 A by LED unit. Figure 1 displays the picture of the LEDs array blanket. In fact, it can be observed in the respective picture (Fig. 1) the blanket with the LED array connected to the power supply (on the right side).

\subsection{Optical characterization of the equipment}

Measurements of the spectral irradiance emitted by the LEDs array were carried out through a spectroradiometer USB from the Ocean Optics Inc. (USA), which presents a spectral response range between 339 and $1019 \mathrm{~nm}$. The distance between the light source (the blanket with the array of LEDs) and the detector (disposed at the center of the illuminated area) was increased by $10 \mathrm{~cm}$ steps, from $10 \mathrm{~cm}$ up to a maximum of $40 \mathrm{~cm}$ away. This last distance corresponds to the average distance recommended for neonatal treatment, when conventional equipments of phototherapy (day-light fluorescent lamps) are employed. A representation of the experimental setup for spectral irradiance measurement as a function of the distance from the blanket is displayed in Fig. 2. This figure demonstrates the blanket with the LEDs array as well as the head of the spectroradiometer, which is being moved along an axis centered in the respective array.

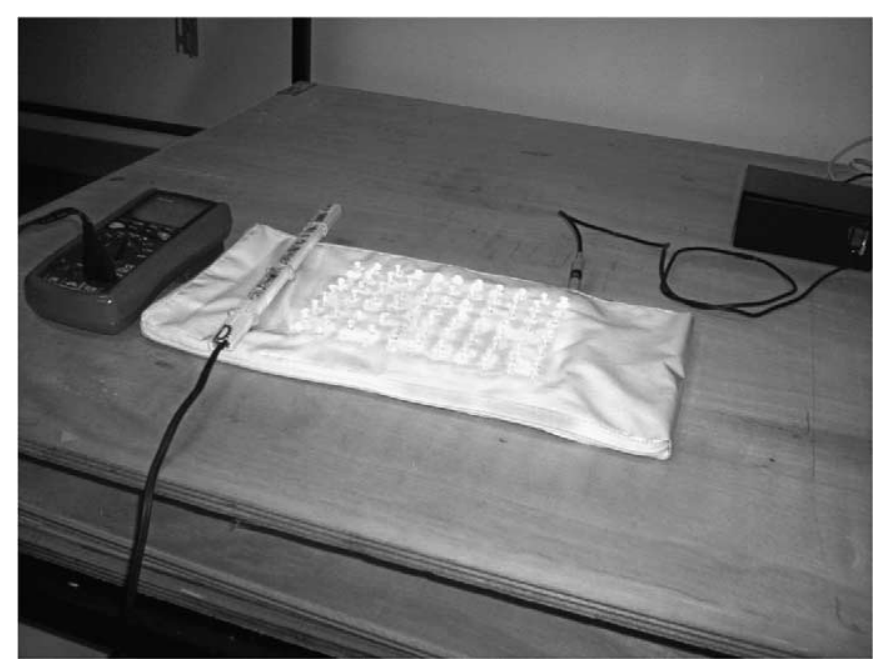

Fig. 1. Picture showing the LEDs array blanket for neonatal phototherapy treatment. 


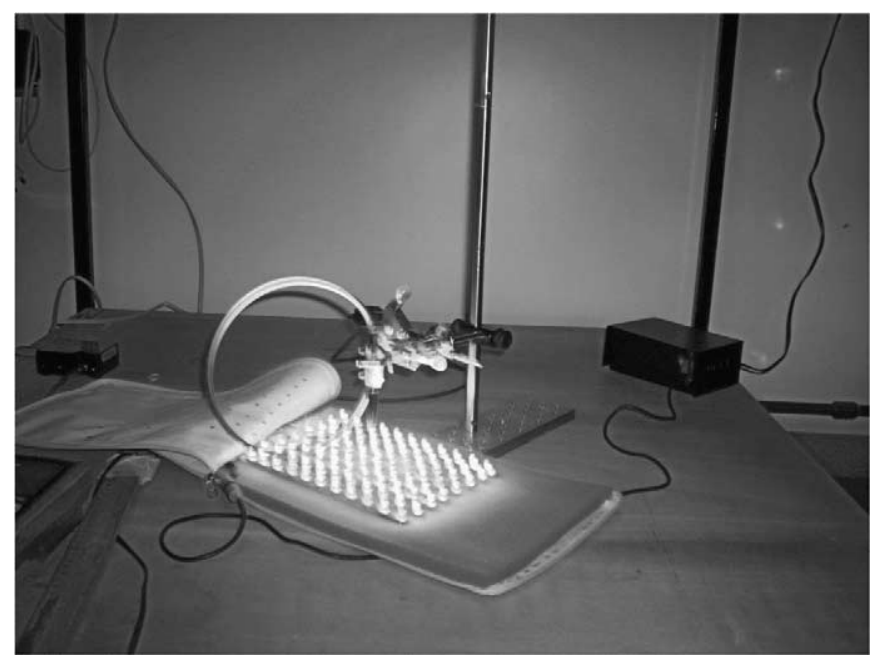

Fig. 2. Experimental setup for spectral irradiance measurement as a function of the distance from the blanket.

The spatial distribution of the irradiance on different parallel planes away from the blanket was determined. It was used a Fluke 189 true RMS Multimeter as well as a radiometer, which was especially build for the experiments of this work at the Instituto de Pesquisas Espaciais (INPE) localized in the city of São José dos Campos, SP, Brazil. The radiometer sensor was a silicon solar cell, which was calibrated regarding its response at $472 \mathrm{~nm}$. The cell had a shunt resistance that converted the current in the solar cell into a specific voltage, which was proportional to the respective irradiance. Irradiance values that were evaluated in this analysis consisted in an average over the illuminated area identified by the detector, which corresponded to its entrance aperture, approximately $2 \mathrm{~cm}$ of diameter. Measurements were taken on planes separated by a distance of $10 \mathrm{~cm}$ to each other, up to a maximum of $40 \mathrm{~cm}$ from the blanket.

In order to compare the efficiency as phototherapy equipment in clinical treatments in relation to other equipments commercially available, we used a conventional device with fluorescent lamps from the Pró-Infância Hospital (São José dos Campos, SP, Brazil). This equipment was fabricated by FANEM, consisting of five $20 \mathrm{~W}$ fluorescent lamps, being two blue-light and three day-light lamps. The optical characterization of this conventional equipment was developed employing the same spectroradiometer USB from the Ocean Optics Inc. (USA), in agreement with previous description.

\subsection{In vivo animal experimentation to comparison of phototherapy efficiency between novel and conventional devices}

\subsubsection{Animals}

Animal care was developed in accordance with the guidelines of the Brazilian College for Animal Experimentation (COBEA), being approved by the Committee for Ethics in Animal Research of UNIVAP (Universidade do Vale do Paraiba, SP, Brazil), receiving register number A054/2007/CEP. Adult male Wistar rats weighting approximately $500 \mathrm{~g}$ ( 3 months old) were used in the respective experimental procedures. Animals were kept in plastic cages, being maintained continuously with water and food $a d$ libitum. The rats were maintained under controlled temperature $\left(26^{\circ} \mathrm{C}\right)$ and on a $12 \mathrm{~h} \mathrm{light/dark}$ cycle. In all, 30 rats, which were randomly divided into 3 groups of 10 animals each, were analyzed in these experiments. Each group was once more divided in two equally subgroups $(n=5)$. 
Hyperbilirubinemia induced by the LCBD technique.

In order to provoke hyperbilirubinemia, the animals received intramuscular administration of two specific drugs. Indeed, $0.1 \mathrm{ml}$ of Ketamine Chloridrate 10\% (Syntec) and Xylazine Chloridrate 2\% (Syntec) were administrated for each $100 \mathrm{~g}$ of corporeal weight of each animal [5,21]. After asepsis in the region of the incision, which was made with iodized alcohol $2 \%$, the surgery was carried by means of medium laparotomia (medium incision of approximately $1.5 \mathrm{~cm}$ ). After the identification process of the biliary duct, it was elaborated a ligation through a surgery wire of non-absorbable suture, which was constituted by a unique filament of polypropylene 6-0. The abdominal wall was closed with simple suture in two plans, using a polypropylene 4-0 surgery wire.

\subsubsection{Experimental procedure}

The thirty animals analyzed in the experiment were randomly divided into six groups of five animals each, according to the following protocol:

(i) Control: negative control, without LCBD surgery;

(ii) LCBD: positive control, with LCBD surgery, without phototherapy treatment.

Serum bilirubin concentration of animals from the two groups, control and LCBD, was evaluated seven days after the initial experimental procedure. Subsequently, they were euthanized.

(iii) LED-8h: animals were irradiated with the LEDs equipment during $8 \mathrm{~h}$;

(iv) LED-24h: animals were irradiated with the LEDs equipment for $24 \mathrm{~h}$;

(v) FLUO-8h: animals were exposed to the conventional fluorescent lamp equipment for $8 \mathrm{~h}$;

(vi) FLUO-24h: animals were exposed to the conventional fluorescent lamp equipment for $24 \mathrm{~h}$.

All phototherapy treatments initiated after the 7th day from the LCBD surgery procedure. Animals were euthanized and the blood bilirubin level was determined immediately after the final step of the phototherapy treatment of each group.

For the phototherapeutic procedure, animals were housing in plastic cages, especially designed for the present experiment, being that two equipments were located at different distances above the animal dorse area. In fact, $10 \mathrm{~cm}$ was the distance chosen for the novel prototype, while $45 \mathrm{~cm}$ was employed for the conventional fluorescent lamps equipment. This last distance was determined in accordance to the international safety rules on neonatal phototherapy treatment that makes use of fluorescent lamp sources.

To blood analysis, it was collected $1 \mathrm{ml}$ of this biological fluid of each animal from the inferior vena cava. After this, the sample was centrifuged, employing Macro III centrifuge, in order to separate the blood serum.

The bilirubin was dosed through diazotation and formation of red azobilirrubin with maximum absorption at $525 \mathrm{~nm}$. The direct bilirubin was dosed in aqueous medium, while that the total bilirubin, including direct and indirect ones, was determined by means of a surfactant of catalyst action. To the procedure of manual preparation, which developed through chemical reactions, were utilized three large assay tubes and one small assay tube for the diazo reactive preparation, in agreement with following procedure:

The diazo reactive (small assay tube) was obtained through a chemical process, in which a drop of nitrite of sodium is mixed to $1.5 \mathrm{ml}$ of sulfanilic acid.

Preparation of large assay tubes for bilirubin test: The assay tubes are called of C (Control), D (direct bilirubin) and $\mathrm{T}$ (total bilirubin). To the assay tube $\mathrm{B}, 2.5 \mathrm{ml}$ of deionized water, $0.2 \mathrm{ml}$ of sulfanilic reactive and $0.05 \mathrm{ml}$ of serum of the blood sample were added. 
To the assay tube $\mathrm{D}, 2.5 \mathrm{ml}$ of deionized water, $0.2 \mathrm{ml}$ of diazo reactive and $0.05 \mathrm{ml}$ of serum originated from the sample. To the assay tube T, $2.5 \mathrm{ml}$ of developer compound, $0.2 \mathrm{ml}$ of diazo reactive and $0.05 \mathrm{ml}$ of serum from the sample. After $5 \mathrm{~min}$, the reactions were analyzed by a Spectrophotometer (Intertek, Stat Fax 3300) at $530 \mathrm{~nm}$.

In this assay, only the total and direct bilirubins were identified. The indirect bilirubin (IB) was obtained through the difference between the total bilirubin (TB) and the direct bilirubin (DB) (IB = TB - DB).

In a specific moment determined for each protocol, the animals were sacrificed. They were anesthetized as described previously, and, after this, a blood sample was taken from the inferior vena cava. This blood sample was utilized for bilirubinemia tests and, then, the animals were euthanized with an overdose of $\mathrm{KCl}$, employing a solution with concentration of $19.1 \%$ (Equiflex).

\subsubsection{Statistical analysis}

Results are expressed as the mean \pm SEM values. The GraphPad Instat v3.0 ${ }^{\circledR}$ software was used for statistical analysis. In order to establish whether the difference between the mean values of two experimental groups was significant, the Student's $t$-test was performed, using a statistical significance level of $p<0.05$.

\section{Results}

Figure 3 displays the spectral irradiance emitted by the array of LEDs as a function of the wavelength, determined at distances of 10,20, 30 and $40 \mathrm{~cm}$, between the luminous source (array of LEDs) and the spectroradiometer head. It was found that for a distance of $10 \mathrm{~cm}$ the spectral irradiance corresponds to a maximum intensity, reaching the value of $77.0 \mu \mathrm{W} / \mathrm{cm}^{2} / \mathrm{nm}$ at the peak of $472 \mathrm{~nm}$, with a FWHM of $50 \mathrm{~nm}$. This value is higher than that obtained from any other phototherapy systems that make use of

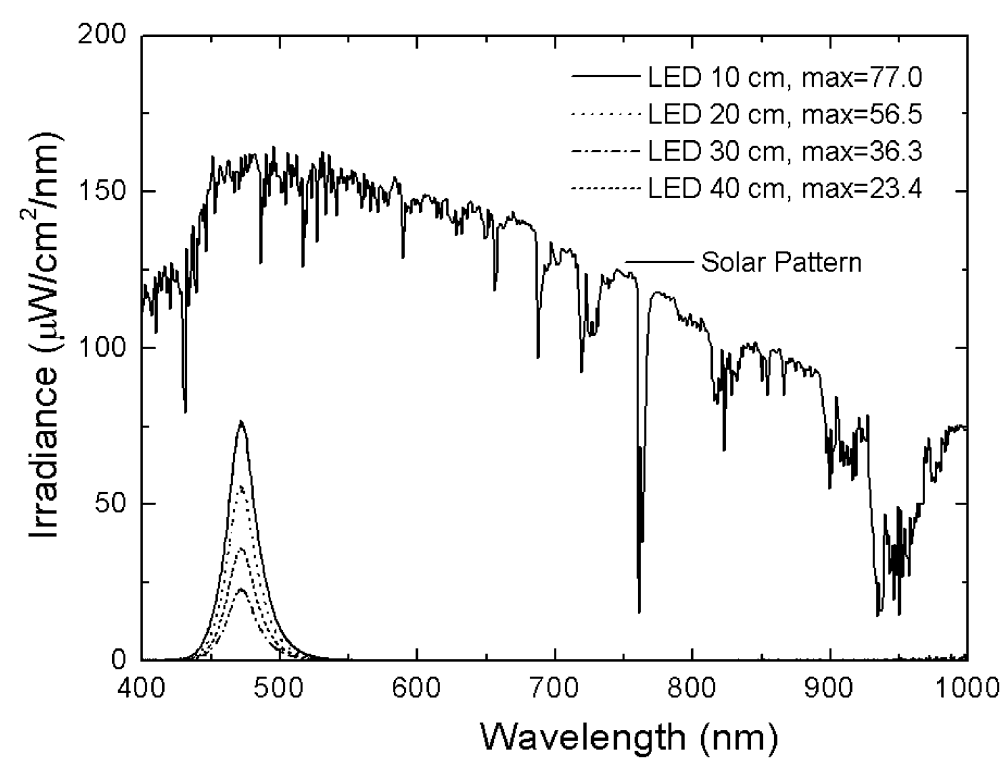

Fig. 3. Irradiation of rats with hyperbilirubinemia using the conventional fluorescent lamps equipment. Spectral distribution of the irradiance emitted by the LEDs blanket light source at distances from 10 to $40 \mathrm{~cm}$. 


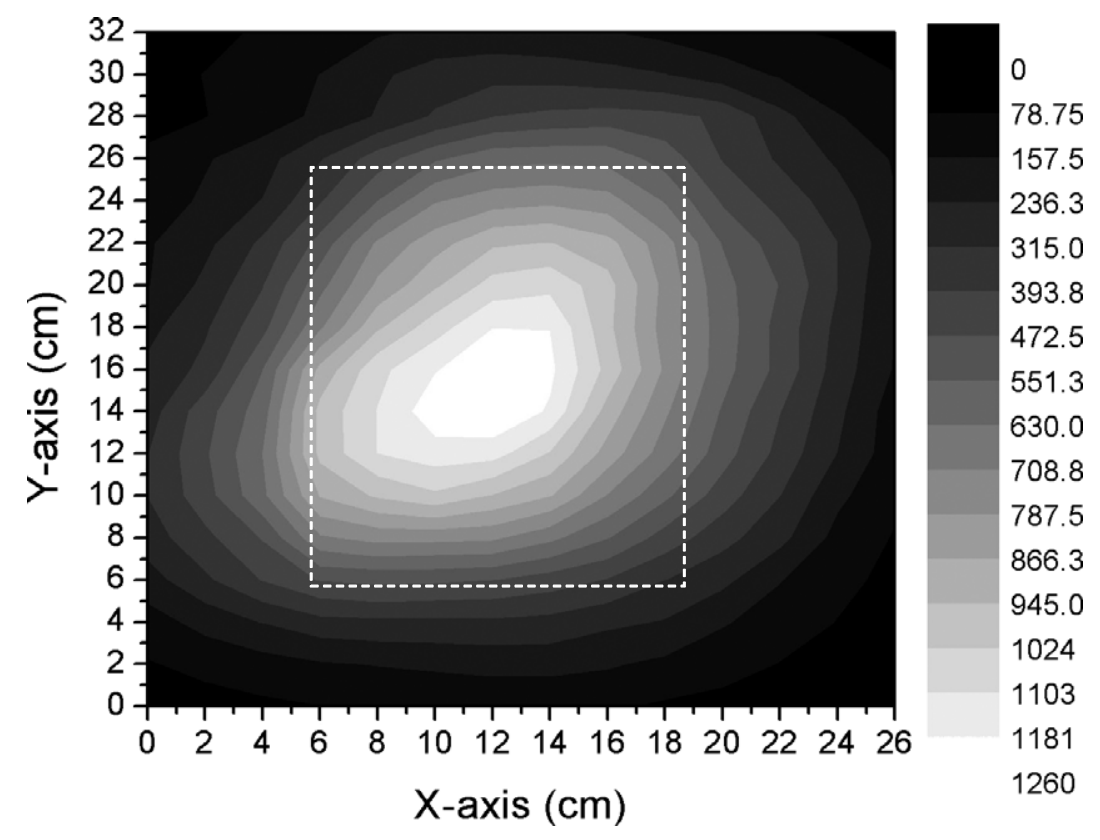

Fig. 4. Spatial distribution of the radiation emitted by the LED's blanket light source over a surface located at $10 \mathrm{~cm}$ from the blanket. The sidebar with the gray tons stands for the incident irradiance in $\mu \mathrm{W} / \mathrm{cm}^{2}$. Dashed line contour represents an area equal to the blanket area $(14 \times 20 \mathrm{~cm})$.

fluorescent or halogen lamps. For instance, we obtained for the fluorescent lamp equipment a maximum spectral irradiance of $5.7 \mu \mathrm{W} / \mathrm{cm}^{2} / \mathrm{nm}$ at the distance of $45 \mathrm{~cm}$, presenting a broad band spectrum that covers the spectral range from 420 to $600 \mathrm{~nm}$.

The spatial distribution of the irradiance at a distance of $10 \mathrm{~cm}$ for the LEDs equipment is shown in Fig. 4. Data represent irradiance values averaged over the illuminated area registered by the detector. It is observed that a maximum intensity occurs at the center of the illuminated area, reaching a value of $1,260 \mu \mathrm{W} / \mathrm{cm}^{2}$. For regions away from the center, the irradiance decreases up to approximately $472.5 \mu \mathrm{W} / \mathrm{cm}^{2}$ around the borderline of an area equal to the blanket size. This value is still very high when compared to that obtained from any conventional phototherapy equipments.

Figure 5 displays the concentration of total bilirubin for each experimental group. It can be observed that the total bilirubin concentration (TB) for the group with LCBD surgery increases by $1,730 \%$ after 7 days from the surgery in comparison with the group without surgery (negative control group). Phototherapy produces a significant reduction of the TB concentration for the LED-8h, LED-24h and FLUO-24h groups, while no significant difference is observed for the FLUO-8h group. The present data demonstrates that phototherapy is capable to reduce the TB levels by $25 \%, 90 \%$ and $30 \%$ for the LED8h, LED-24h and FLUO-24h groups, respectively. The phototherapy treatment during $24 \mathrm{~h}$, through the new LEDs equipment showed to be more efficient than the other phototherapy protocols. Indeed, the TB concentration reduction by LED-24h is $90 \%$ higher than that from LED-8h and FLUO (either 8 or $24 \mathrm{~h}$ irradiation time).

The direct bilirubin (DB) serum concentration for all the experimental groups is shown in Fig. 6 . As for the case previously discussed, the LCBD surgery causes a large increment regarding the BD concentration $(1,170 \%)$, seven days after the surgery procedure. Phototherapy treatment with LEDs equipment during $8 \mathrm{~h}$ as well as with the conventional fluorescent lamps equipment for 8 or $24 \mathrm{~h}$ does 

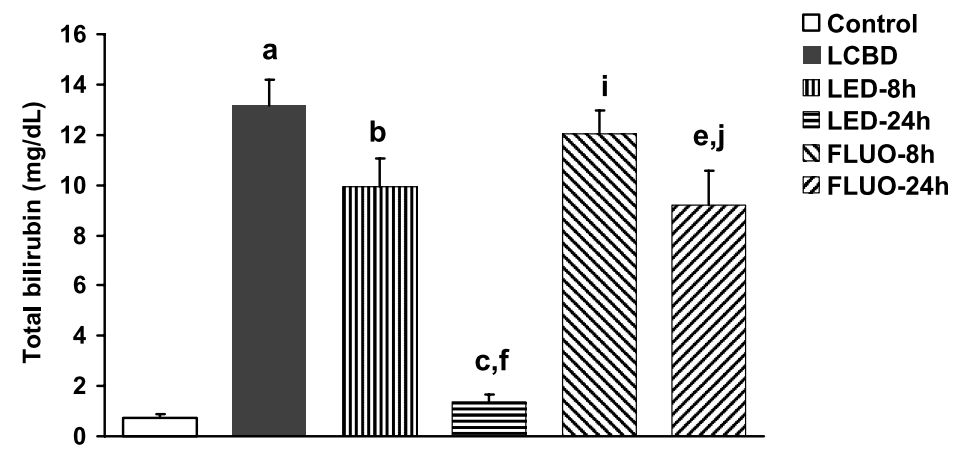

Fig. 5. Serum concentration of the total bilirubin for the experimental groups. (a) LCBD vs. control: $p<0.001$; (b) LED-8h vs. LCBD: $p<0.05$; (c) LED-24h vs. LCBD: $p<0.001$; (e) FLUO-24h vs. LCBD: $p<0.05$; (f) LED-24h vs. LED-8h: $p<0.001$; (i) FLUO-8h vs. LED-24h: $p<0.001$; (j) FLUO-24h vs. LED-24h: $p<0.001$.

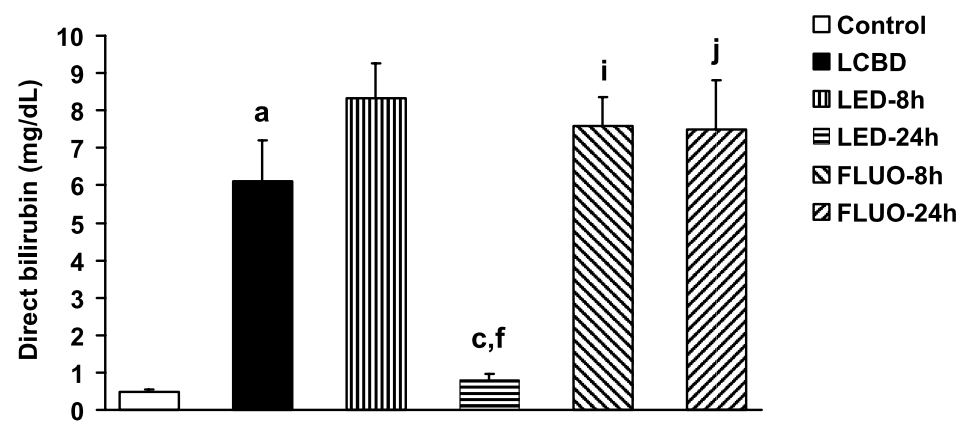

Fig. 6. Serum concentration of the direct bilirubin for the experimental groups. (a) LCBD vs. control: $p<0.001$; (c) LED-24h vs. LCBD: $p<0.01$; (f) LED-24h vs. LED-8h: $p<0.001$; (i) FLUO-8h vs. LED-24h: $p<0.01$; (j) FLUO-24h vs. LED-24h: $p<0.01$.

not demonstrate significant effect on the DB concentration. On the other hand, the treatment with the new prototype during $24 \mathrm{~h}$ causes a significant reduction of the DB level by an amount as high as $87 \%$.

Although the level of indirect bilirubin (IB) can be calculated as the difference between TB and DB, it is interesting to determine the values of IB separately, since the neonatal jaundice is directly related to the increase of the IB concentration. In fact, with this procedure, the precision of the respective methodology would be significantly improved. For this reason, we display the IB concentration for all the animal groups with and without phototherapy treatment (Fig. 7).

Figure 7 demonstrates that LCBD surgery is quite efficient to induce bilirubinemia, increasing pronouncedly the IB concentration by $2,840 \%$. It is also found that all the phototherapy protocols investigated constitute suitable methodologies to reduce the jaundice. This can be inferred due to the significant photodegradation of the IB molecules, which is evidenced by the values of $77 \%, 92 \%, 35 \%$ and $76 \%$, for groups LED-8h, LED-24h, FLUO-8h and FLUO-24h, respectively. In this context, it is important to notice that the phototherapy with the novel LEDs equipment during $24 \mathrm{~h}$ seems to be the most efficient for jaundice treatment. If the irradiation time interval is lengthening from 8 to $24 \mathrm{~h}$, the IB concentration decays from $23 \%$ to $8 \%$, when compared with its respective value before the phototherapy treatment. In order to produce the same IB concentration reduction (76\% for FLUO-24h and 77\% for LED-8h), the conventional procedure, which uses the Fluorescent lamps equipment, needs to be approximately three 


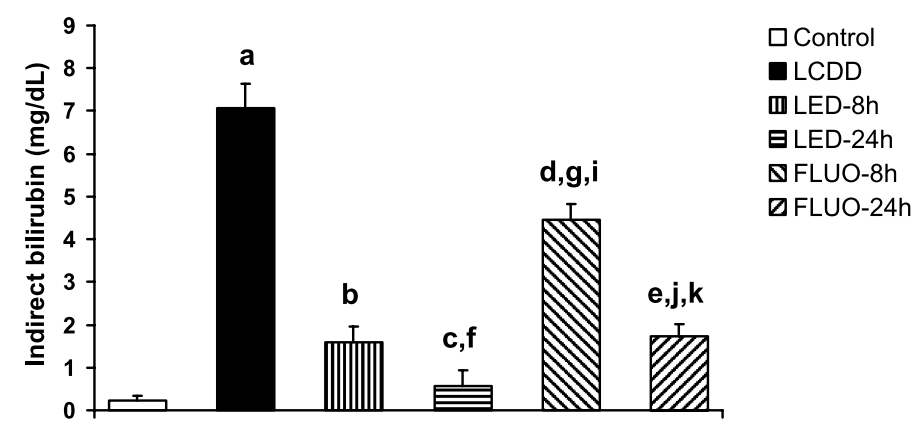

Fig. 7. Serum concentration of the indirect bilirubin for the experimental groups. (a) LCBD vs. control: $p<0.001$; (b) LED-8h vs. LCBD: $p<0.001$; (c) LED-24h vs. LCBD: $p<0.001$; (d) FLUO-8h vs. LCBD: $p<0.001$; (e) FLUO-24h vs. LCBD: $p<0.001$; (f) LED-24h vs. LED-8h: $p<0.005$; (g) FLUO-8h vs. LED-8h: $p<0.001$; (i) FLUO-8h vs. LED-24h: $p<0.05$; (j) FLUO-24h vs. LED-24h: $p<0.05$; (k) FLUO-24h vs. FLUO-8h: $p<0.001$.

times longer than for the LEDs equipment. This representative difference demonstrates the efficacy of the new device.

\section{Discussion}

The therapeutic efficiency of any neonatal phototherapy equipments depends on the serum bilirubin concentration in the patient before to initiate the treatment, wavelength of the light source, distance between the patient and the luminous source, irradiance dose and corporal area exposed to the light. Besides of that, it is important to mention also the intrinsic characteristics of the neonates, such as weight, nutrition status and associated pathologies [10]. Actually, several light sources are being applied, but these devices present some advantages as well as some drawbacks, which can affect decisively the efficiency of the phototherapy. For instance, the light from a fluorescent lamp can irradiate a large corporal area of the neonate, but its radiance is low and the emission spectrum is very broad, i.e., with a pronounced polychromatic character, covering the spectral range from 380 to $770 \mathrm{~nm}$. However, the bilirubin absorbs only radiation between 400 and $500 \mathrm{~nm}$, implying that all the incident photons of wavelength lower than $400 \mathrm{~nm}$ and higher than $500 \mathrm{~nm}$ cannot be absorbed by the bilirubin compound. In this way, the photons of the spectral ranges between 380 and $400 \mathrm{~nm}$ as well as from 500 up to $770 \mathrm{~nm}$ are converted in heat. For this reason, fluorescent lamps should be located at large distances from the patient, approximately between 30 and $50 \mathrm{~cm}$, in order to avoid an overheating process.

In this context, it is important to register that the light from halogen lamps, which is known as the "special blue light", was introduced in clinical practice in 1972 for neonatal phototherapy. Several works have demonstrated that the special blue light produces faster and higher reduction of the serum bilirubin concentration as compared to the traditional systems using fluorescent white light. Systems with halogen lamps present an excellent spectral irradiance, between 25 and $35 \mu \mathrm{W} / \mathrm{cm}^{2} / \mathrm{nm}$; but, they cannot be situated in a position very close to the neonates due to overheating and burning problems, being recommended a distance of $40-50 \mathrm{~cm}$ from the patient. Although halogen systems can illuminate an area of $20 \mathrm{~cm}$ of diameter, its irradiance presents higher intensity only at the center of this region. Consequently, they are efficient for small neonates (up to $1,500 \mathrm{~g}$ of weight), but quite inefficient for bigger patients (2,500 g or more).

Since the phototherapy acts thought the patient skin, it is possible to conclude that the extension of the corporal area exposed to the light is also an important parameter for the treatment efficiency. 
As larger the area and closer the light source in relation to the patient, more efficient should be the phototherapy treatment. The irradiance for conventional equipment using fluorescent lamps increases from $4 \mu \mathrm{W} / \mathrm{cm}^{2} / \mathrm{nm}$ for a distance of $30 \mathrm{~cm}$ up to $12 \mu \mathrm{W} / \mathrm{cm}^{2} / \mathrm{nm}$ when the distance is reduced to $10 \mathrm{~cm}$ from the patient. However, with this great proximity, it can occur overheating and dehydration of the neonate. Usually, a conventional system with fluorescent lamps is positioned between 30 and $40 \mathrm{~cm}$ from the patient; whereas, the one that uses halogen lamps is maintained at $50 \mathrm{~cm}$ in order to avoid the above mentioned negative effects.

To the validation and analysis of the new LEDs system, hyperbilirubinemia was induced by surgery procedure of obstruction of the biliary duct of Wistar rats. This procedure results in an accumulation of substances that normally are eliminated by the biliary ducts. Indeed, the jaundice induced by obstruction of the biliary duct generates several disturbs like, for example, the accumulation of toxic substances (indirect bilirubin), mainly due to the enterohepatic circulation increase, which is associated to a great activity of the beta-glucuronidase enzyme. This biochemical process originates the hydrolysis of the conjugated bilirubin, propitiating a new formation of the indirect reaction pigment. Subsequently, this compound is reabsorbed in the intestinal mucosa, returning to the systemic circulation to generate a higher concentration of the total bilirubin (Fig. 5).

We have developed preliminary experimental studies focused on the bilirubinemia induced in rats by the LCBD technique. These analyses have showed a continuous and significant increase of the bilirubin concentration with time after the surgery, reaching the hyperbilirubinemia state at the seven days after surgery. For this reason, we started all the phototherapy treatment experiments at that specific time. LCBD technique proved to be very efficient to induce the hyperbilirubinemia in rats, producing serum bilirubin concentration as high as: TB $-13.18 \mathrm{~g} / \mathrm{dl}$, DB $-6.12 \mathrm{mg} / \mathrm{dl}$ and IB $-7.06 \mathrm{mg} / \mathrm{dl}$.

It was observed that phototherapy treatment for $8 \mathrm{~h}$ using fluorescent lamps equipment did not reduce significantly the serum bilirubin concentration in animals with LCBD. Probably, these results indicate that conventional phototherapy could be inefficient for short time hyperbilirubinemia treatment.

Phototherapy treatments LED-8h, FLUO-8h and FLUO-24h were not significant to reduce the serum concentration of direct bilirubin in rats with LCBD; whereas that LED-24h treatment demonstrated to be highly effective. This fact means that LED therapy could be also an auspicious candidate to treat other pathologies associated to the direct bilirubin.

Analysis of data shown in Figs 6-8 indicates that to obtain the same effect on serum concentration of total (direct and indirect) bilirubin, the conventional phototherapy methodology requires much more time to light interaction. In fact, in order to reach an optimum result, the fluorescent lamps equipment, instead of our LED equipment, needs to increase the time of treatment in up to three times, like, for example, from 8 to $24 \mathrm{~h}$.

Therefore, results on serum indirect bilirubin levels demonstrate that phototherapy using LEDs is more efficient than fluorescent lamp for the same time of treatment. Irradiation with light from LEDs during $24 \mathrm{~h}$ causes a decrease of the indirect bilirubin serum concentration as high as $92 \%$.

Chang et al. in 2005 [6] reported that phototherapy with LEDs devices irradiation provokes a faster degradation of the bilirubin molecules when compared with the conventional light sources. They demonstrated around $30 \%$ bilirubin degradation when irradiating Gunn rats with their LEDs device during $5 \mathrm{~h}$ at a spectral irradiance of $75 \mu \mathrm{W} / \mathrm{cm}^{2} / \mathrm{nm}$. In our experiment, we obtained a bilirubin degradation of: TB-25\% and IB-90\% for $8 \mathrm{~h}$ irradiation and TB-90\% and IB-92\% for $24 \mathrm{~h}$, at a spectral irradiance of $77 \mu \mathrm{W} / \mathrm{cm}^{2} / \mathrm{nm}$, which is very close to that used by Chang et al. [6]. It is worthy to mention that both experiments were carried out in two different animal models: Gunn rats in Chang's work and rats with hyperbilirubinemia induced by LCBD surgery in the present article. 
These results and their respective methodologies must be considered in order to reduce quickly serum bilirubin levels, which is characteristic biochemical profile of jaundice neonates. These new possibilities can avoid the need for blood transfusion, reducing on neonates the risk for the neurologic syndrome known as Kernicterus, which can lead to severe neurologic sequels and even death [3].

Further tests of the equipment performance are required to its development before it can be used for clinical applications. New experiments of this prototype, which soon will be published, are been made and preliminary results seem to be very auspicious.

\section{Conclusion}

The characterization of a new device developed by our group indicates that this LEDs equipment presents several very interesting properties that become this prototype an excellent candidate to applications in clinical procedures. Indeed, the novel equipment presents positive characteristics, such as a large and uniform area of irradiation, efficient wavelength emission for jaundice treatment, high spectral irradiance, simplicity, low weight, low heating and low cost. Furthermore, tests on an animal model showed that our LED-therapy equipment is more efficient for bilirubin molecule degradation than the conventional phototherapy, i.e., the phototherapic procedure that uses fluorescent lamps equipments commercially available. Finally, it can be concluded that the developed equipment can be a very promising candidate for neonates' phototherapy treatment.

\section{Acknowledgements}

P.F. Sebbe acknowledges CAPES - Coordenação de Aperfeiçoamento de Pessoal de Nível Superior (Brazil) for the scholarship that made possible this work. Authors also acknowledge MD Márcia Elisa S. de Oliveira Ramos (Laboratory of Clinical Exams "Antônio Afonso de Jacareí, SP, Brazil"), the Pró-Infância Hospital of São José dos Campos, SP, Brazil, for lending us the phototherapy fluorescent equipment, and MD Cláudio S. Menêses from CIPAX (Center for Diagnostic Medicine, Pathology and Cytology), São José dos Campos, SP, Brazil.

\section{References}

[1] M. Amato and D. Inaebnit, Clinical usefulness of high intensity green light phototherapy in the treatment of neonatal jaundice, Eur. J. Pediatr. 150 (1991), 274-276.

[2] H. Ayyash, E. Hadjigeorgiou, I. Sofatzis, H. Dellagrammaticas and E. Sideris, Green or blue light phototherapy for neonates with hyperbilirubinemia, Arch. Dis. Child 62 (1987), 843-845.

[3] M.A. Brito, R.F.M. Silva and D. Brites, Bilirubin toxicity to human erythrocytes: A review, Clin. Chim. Acta 374 (2006), 46-56.

[4] G.R. Cameron and C.L. Oakley, Ligation of the common bili duct, J. Pathol. Bact. 35 (1932), 769-798.

[5] M. de Carvalho, D. de Carvalho, S. Trzmielina, J.M.A. Lopes and T.W.R. Hansen, Intensified phototherapy using daylight fluorescent lamps, Acta Pediatr. 88 (1999), 768-771.

[6] Y.S. Chang, J.H. Hwang, H.N. Kwon, C.W. Choi, S.Y. Ko, W.S. Park, M.S. Shin and M. Lee, In vitro and in vivo efficacy of new blue light emitting diode phototherapy compared to conventional halogen quartz phototherapy for neonatal jaundice, J. Korean Med. Sci. 20 (2005), 61-64.

[7] S.T. Chou and J.B. Gibson, A histochemical study of the bili ducts in long term biliary obstruction in the rat, J. Pathol. 103 (1971), 163-175.

[8] A.P. Colvero, M.O. Colvero and R.M. Fiori, Modulus of teaching phototherapy, Scientia Medica 15 (2005), 125-132. 
[9] R.J. Cremer, P.W. Perryman and D.H. Richards, Influence of light on the hyperbilirubinemia of infants, Lancet 1 (1958), 1094-1097.

[10] P. Dicken, L.J. Grant and S. Jones, An evaluation of the characteristics and performance of neonatal phototherapy equipment, Physiol. Meas. 21 (2000), 493-503.

[11] J.F. Ennever, M. Sobel, A.F. Mcdonagh and W.T. Speck, Phototherapy for neonatal jaundice: in vitro comparison of light sources, Pediatr. Res. 18 (1984), 667-670.

[12] J.F. Ennever, Blue light, green light, white light, more light: treatment of neonatal jaundice, Clin. Perinatol. 17 (1990), 467-481.

[13] B.B. Frank, Clinical evaluation of jaundice: a guideline of the patient care committee of the American Gastroenterological Association, J. Am. Med. Assoc. 262 (1989), 3031-3034.

[14] I. Gilmore and C.J. Garvey, Investigation of jaundice, Medicine 31 (2006), 13-16.

[15] L.P. Halamek and D.K. Stevenson, Neonatal jaundice and liver disease, in: Neonatal-Perinatal Medicine: Diseases of the Fetus and Infant, 6th edn, Vol. 2, A.A. Fanaroff and R.J. Martin, eds, Mosby Yearbook, St. Louis, MO, 1997, pp. 1-1345.

[16] J. Lucey, M. Ferreiro and J. Hewitt, Prevention of hyperbilirubinemia of prematurity by phototherapy, Pediatrics 41 (1968), 1047-1054.

[17] M.J. Maisels, Phototherapy - traditional and nontraditional, J. Perinatol. 21(Suppl. 1) (2001), S93-S97.

[18] S. Nakamura, S. Pearton and G. Fasol, The Blue Laser Diode: GaN Based Light Emitters and Lasers, 2nd revised and enlarged edn, Springer, 1997.

[19] H. Rosen, A. Rosen, D. Rosen, B. Onaral and M. Hiatt, Use of a light emitting diode (led) array for bilirubin phototransformation, in: Engineering in Medicine and Biology Society, IEEE-EMBS, 27th Annual International Conference, IEEE-EMBS, New Brunswick, NJ, 2005, pp. 7266-7268.

[20] P.F. Sebbe, A.B. Villaverde, R.A. Nicolau, A.M. Barbosa and N. Veissid, Characterization of an optical device with an array of blue light emitting diodes LEDs for treatment of neonatal jaundice, in: RIAO/OPTILAS 2007, N.U. Wetter and J. Frejlich, eds, Vol. 992, 2008, pp. 606-610.

[21] D.S. Seidman, J. Moise, Z. Ergaz, A. Laor, H.J. Vreman, D.K. Stevenson and R. Gale, A new blue light-emitting phototherapy device: A prospective randomized controlled study, J. Pediatr. 136 (2000), 771-774.

[22] D.S. Seidman, J. Moise, Z. Ergaz, A. Laor, H.J. Vreman, D.K. Stevenson and R. Gale, A prospective randomized controlled study of phototherapy using blue and blue-green light-emitting devices, and conventional halogen-quartz phototherapy, J. Perinatol. 23 (2003), 123-127.

[23] S. Steffensrud, Hyperbilirubinemia in term and near-term infants; Kernicterus on the rise?, Newborn Infant Nurs. Rev. 4 (2004), 191-200.

[24] K.L. Tan, The pattern of Bilirubin response to phototherapy for neonatal hyperbilirubinaemia, Pedriatr. Res. 16 (1982), 670-674.

[25] K.L. Tan, Phototherapy for neonatal jaundice, Clin. Perinatol. 18 (1991), 423-439.

[26] K.L. Tan, Comparison of the efficacy of fiberoptic and conventional phototherapy for neonatal hyperbilirubinemia, $J . P e-$ diatr. 125 (1994), 607-612.

[27] C. Vecchi, G.P. Dozelli and M.G. Migliorini, Green light in phototherapy, Pediatr. Res. 17 (1983), 461-466.

[28] C. Vecchi, G.P. Dozelli, G. Sbrana and R. Pratesi, Phototherapy for neonatal jaundice: Clinical equivalence of fluorescent green and "special" blue lamps, J. Pediatr. 108 (1986), 452-466.

[29] H.J. Vreman, R.J. Wong, J.R. Murdock and D.K. Stevenson, In vitro efficacy measurements of LED-based phototherapy devices compared to traditional light sources in a model system, in: EASL International Bilirubin Workshop, EASL, Trieste, Italy, 2004, pp. 51-56.

[30] H.J. Vreman, R.J. Wong and D.K. Stevenson, Phototherapy: current methods and future directions, Semin. Perinatol. 28 (2004), 326-333.

[31] H.J. Vreman, R.J. Wong, D.K. Stevenson, R.K. Route, S.D. Reader, M.M. Fejer, R. Gale and D.S. Seidman, Light-emitting diodes: A novel light for phototherapy, Pediatr. Res. 44 (1998), 804-809. 


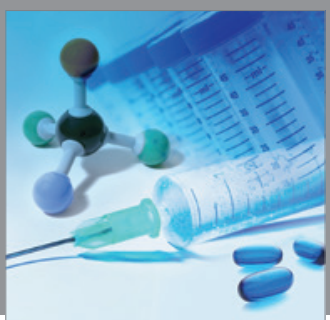

International Journal of

Medicinal Chemistry

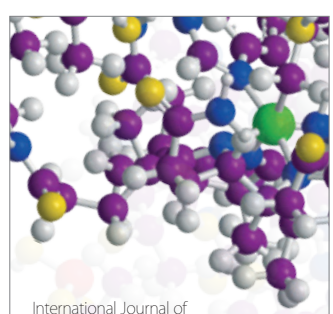

Carbohydrate Chemistry

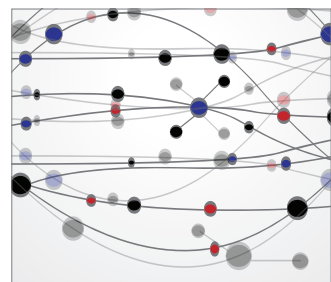

The Scientific World Journal
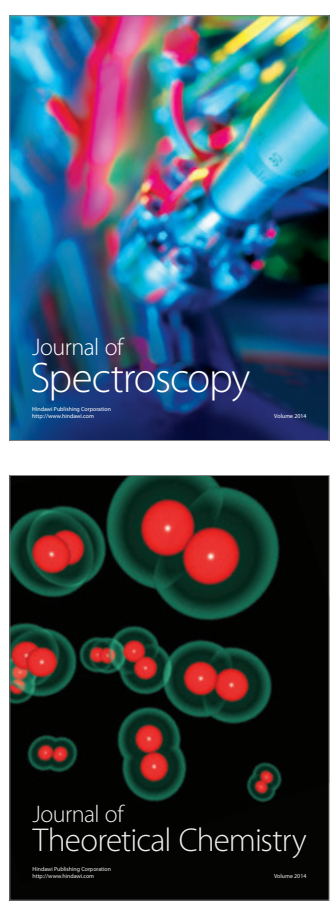
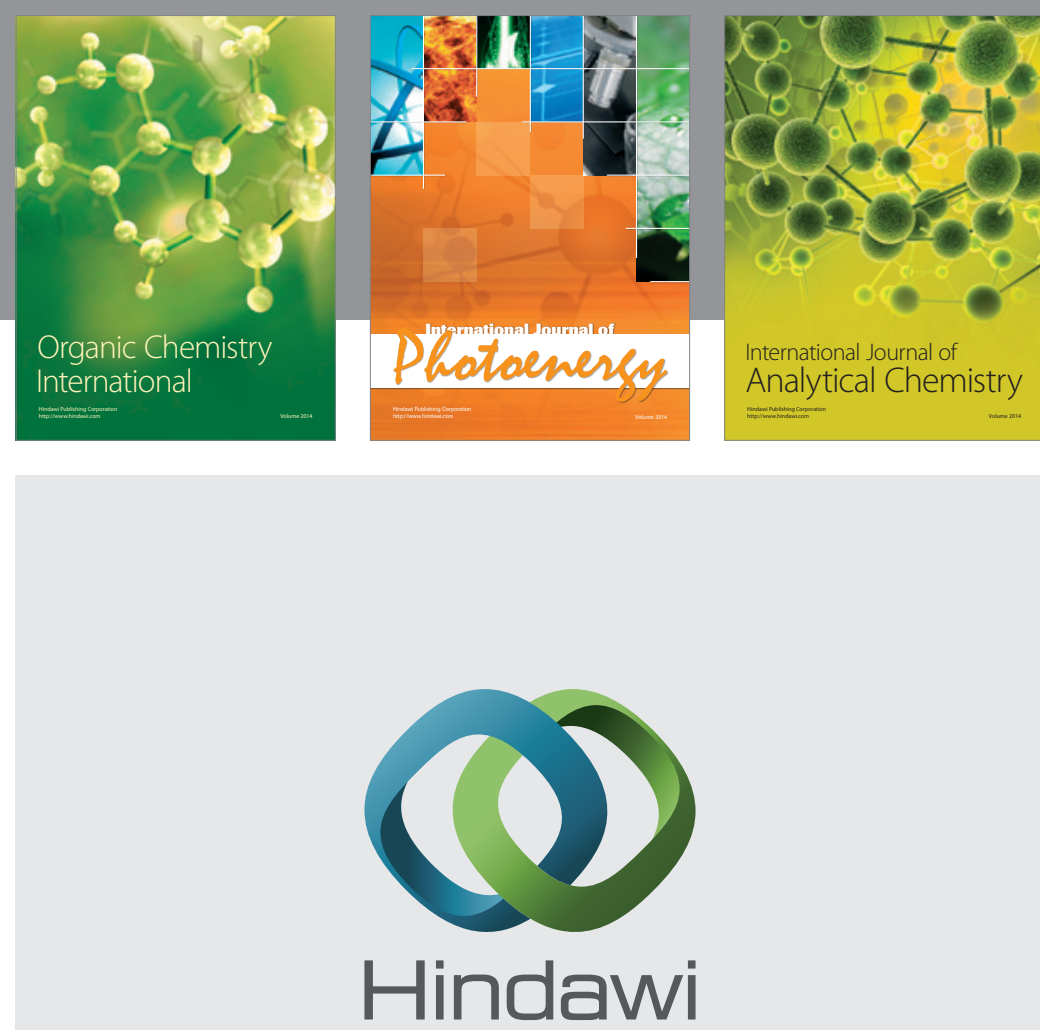

Submit your manuscripts at

http://www.hindawi.com
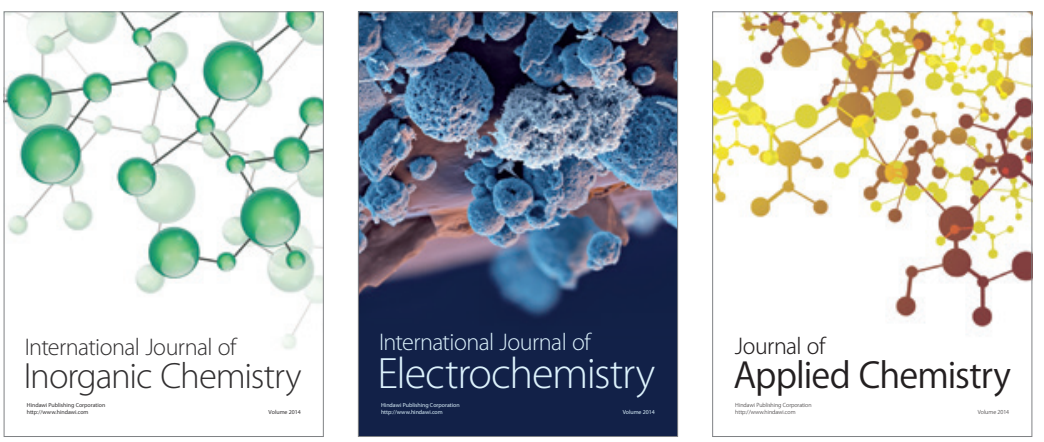

Journal of

Applied Chemistry
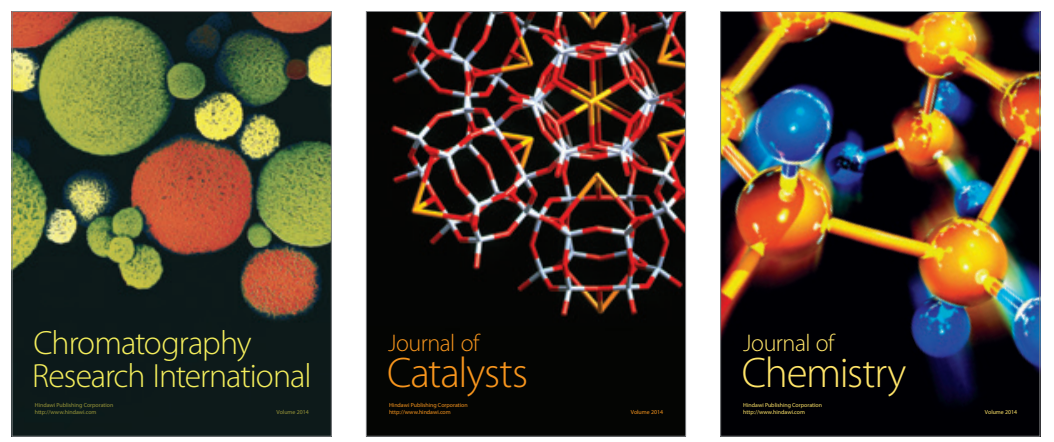
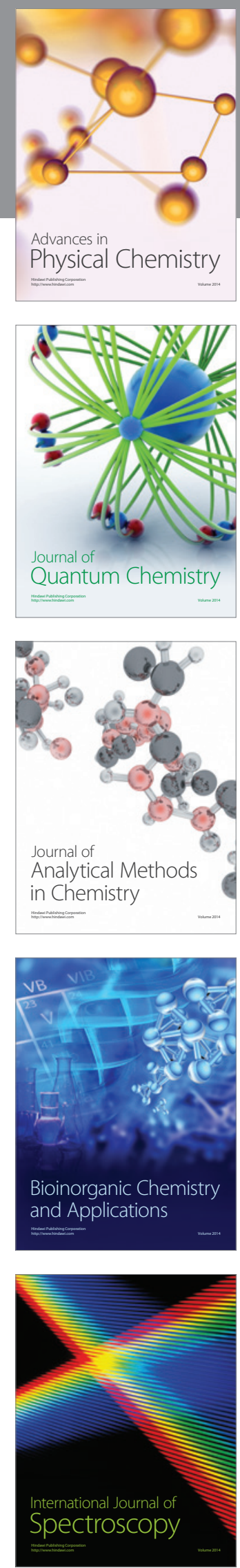